\title{
Bases para el establecimiento de un plan de estudios en geografía regional
}

José María Marín JaIme *

\section{INTRODUCCIÓN}

En la Universidad Española, la perspectiva de unos planes docentes que han de ser renovados en un plazo relativamente corto, es un hecho importante que solicita de la comunidad de geógrafos, particularmente españoles, la reflexión y sistematización de las ideas que han ido surgiendo en el tiempo en que han venido ejerciendo su labor profesional, $y$ el enriquecedor intercambio de experiencias con los geógrafos de otros países.

En las siguientes líneas deseamos exponer nuestra visión personal sobre lo que consideramos puede ser la organización de un plan de ordenación docente en los estudios universitarios de Geografía, deteniéndonos de modo más específico en los elementos necesarios para la formación de profesionales expertos en análisis regionales.

Todo plan de ordenación docente requiere, por una parte, cubrir los objetivos derivados del carácter y naturaleza de la ciencia que estudia, en nuestro caso, la Geografia y, por otra, atender las demandas sociales, siempre variables, que pueden ser satisfechas por la misma.

\footnotetext{
* Dpto. Geografía y Ordenación del Territorio. Universidad de Zaragoza.
} 
Atender al primer aspecto, carácter y naturaleza de la Ciencia Geográfica, es obvio puesto que cada una de las ramas del saber científico tiene sus propios fundamentos conceptuales y metodológicos. Sin embargo, para nosotros los geógrafos, este aspecto tiene especial importancia por la pluralidad de enfoques existentes en la especialidad (Estébanez, 1982). Pluralidad de enfoques que ni deben conducirnos al escepticismo sobre la entidad de nuestra Ciencia, ni suscitar temores acerca del buen hacer del trabajo profesional que realizamos, pero que nos obliga a explicitar la concepción global que tenemos de la Geografía, como paso previo, justificación teórica, metodológica y didáctica de la posterior relación de materias a incluir en nuestro ordenamiento docente.

Prestar atención en nuestro proyecto docente a las demandas sociales de profesionales es algo más que satisfacer la simple necesidad de buscar utilidad a una ciencia tradicionalmente impartida en la Universidad. El ejercicio de la actividad docente no puede obviar el compromiso ante aquellos alumnos que han encontrado en la Geografía una forma de interpretar y comprender el mundo y que se sienten cómodos con las coordenadas conceptuales y metodológicas de la misma; estos alumnos tienen derecho a encontrar una actividad que les permita desarrollar y practicar las habilidades para las que han sido preparados y en ello un medio de vida y de satisfacción personal.

En concordancia con estas premisas, para el propósito que nos guía es conveniente reflexionemos acerca de los siguientes apartados:

- Concepción de la Ciencia Geográfica y del Análisis Geográfico Regional.

- Demandas sociales y la orientación de los estudios, de forma particular, en el área de Análisis Geográfico Regional.

- Líneas directrices en los estudios del área de conocimiento Análisis Geográfico Regional.

Antes, permítasenos un breve inciso sobre la actual división de los Departamentos universitarios españoles en tres áreas de conocimiento.

El proceso de renovación de la Universidad española, puesto en marcha por la Ley de Reforma Universitaria, así como los planes de estudio, ha desembocado en la creación de tres áreas de conocimiento asignadas a los Departamentos de Geografía: Geografía Física, Geografía Humana y Análisis Geográfico Regional.

Desconocemos hasta que punto han sido motivaciones epistemológicas las que han aconsejado la creación de estas tres áreas; como 
siempre ocurre en los procesos de cambio instaurados desde la Administración muy probablemente han podido pesar más razones de coyuntura política, sobre las propiamente científicas en el momento de establecer esta división. No obstante, a nuestro parecer, consideramos adecuada la actual distribución por los siguientes motivos:

- Cada una de las áreas tiene objetivos específicos, una metodología de trabajo adecuada a la consecución de dichos objetivos y unos contenidos diferenciados. La delimitación de estos aspectos, objetivos -metodologia - contenidos, puede resultar problemática entre las áreas de Geografia Regional y Geografia Humana si bien, como tendremos ocasión de establecer, a cada una de estas dos áreas compete una función propia y original en el interior de la Ciencia Geográfica.

- Recoge con bastante fidelidad la realidad vigente en los planes de ordenación docente y principales líneas de investigación de gran parte de los Departamentos de Geografía españoles.

De este modo, del corpus tradicionalmente denominado Geografia General se han derivado dos áreas, Geografía Humana y Geografía Fisica, que van a dar lugar a formaciones específicas de los estudiantes recogiendo lo que es una realidad en los estudios geográficos de hoy.

Por su parte el área de Análisis Geográfico Regional acoge las antiguas materias de Geografía Descriptiva, Geografía de Paises, que si bien han perdido interés en los planes de estudio de algunas universidades, sigue teniendo vigencia en otras muchas, aparte de la tradicional vinculación de la Geografía con este tipo de estudios.

- Con las tres áreas se cubren perfectamente las necesidades derivadas de la proyección social que, ya hoy día y esperemos que en el futuro, tienen los estudios geográficos. Formulando coherentemente los planes de estudios de las tres áreas de conocimiento, el geógrafo puede contribuir con otros profesionales al esclarecimiento y aporte de soluciones de los complejos problemas de la sociedad actual.

Sin embargo, admitir la conveniencia e incluso la utilidad de esta división en áreas como medio de favorecer un mayor progreso en los estudios geográficos, no puede hacernos olvidar que hay un sustantivo "Geografía" que acoge la adjetivación corres- 
pondiente a cada una de las áreas y que es en definitiva el que da entidad a la labor de un Departamento de Geografía.

\section{MARCO CONCEPTUAL Y TEÓRICO DEL PLAN DE ORDENACIÓN DOCENTE}

\subsection{Contenidos temáticos de la Ciencia Geográfica}

El campo de trabajo de los profesionales de la Geografia ha oscilado, a lo largo de la historia, en torno a un número relativamente reducido de temas los cuales conforman las tradiciones temáticas básicas de nuestra disciplina.

Capel (1982), recogiendo las conclusiones de aquellos estudios que han trabajado esta materia y en función de las principales definiciones que de la Geografía se han dado, opina que pueden establecerse seis tradiciones temáticas en Geografía entre las cuales, a su vez, existen asociaciones que permiten reducir únicamente a dos, lo que Capel denomina "problema-clave", los temas que han interesado y dado continuidad a nuestra disciplina en el tiempo.

Las seis tradiciones temáticas establecidas por Capel son: Física, Corológica, Ecológica, Paisajística, Espacial y Social. Los problemasclave identificados, una vez que se establecen asociaciones entre las distintas tradiciones temáticas, hacen referencia a la «diferenciación del espacio en la superficie terrestre" y a la "relación entre el hombre y el medio" (Capel, 1982).

Conocimiento del espacio y sentido integrador son dos aspectos, a mi juicio, determinantes del quehacer geográfico. En ellos vamos a detenernos brevemente a continuación.

\subsubsection{Dimensión espacial de los estudios geográficos}

De los distintos planos en que puede articularse el conocimiento sobre la realidad espacial, la Ciencia Geográfica ha puesto su interés: 
- por una parte en el estudio, explicación y valoración de todo aquello que tiene significación espacial, bien porque aparece como contenido o propiedad del espacio (espacio dado), bien en la medida en que se constituye en agente que configura y organiza el espacio (espacio producido; espacio social);

- por otra, mediante el análisis y reconocimiento de áreas espaciales concretas.

El espacio geográfico es en primer lugar, aunque no prioritariamente, un espacio dado por cuanto aparece compuesto por un conjunto de elementos físicos y humanos e informado en un momento dado por características económicas concretas. En el análisis que del espacio realiza, el geógrafo toma en consideración uno o varios de estos elementos, indicando sus características, las formas bajo las que se presenta, los lugares en que se localiza, las pautas de su distribución, las causas y consecuencias que se derivan de su presencia o ausencia, los factores que han condicionado su evolución...

Numerosos aspectos del medio natural: formas de relieve, tipos de clima, recursos hídricos superficiales... son abordados en los trabajos realizados por geógrafos como elementos que definen, más allá de la mera localización y distribución de los mismos, la realidad de distintos espacios, permitiendo establecer conceptos, tipologias clasificatorias y leyes que rigen su funcionamiento.

De igual manera es normal que la tarea del geógrafo profundice y se interese por el espacio en cuanto que éste acoge al hombre y sus actividades. Caracteristicas evolutivas y actuales de diversos grupos sociales asentados en un territorio, conocimiento de las prácticas económicas por ellos realizadas, situación y nivel de desarrollo del conjunto de la actividad económica, asi como de cada uno de los sectores... han formado y forman parte de las preocupaciones geográficas. También en este campo de estudio se deducen teorias y leyes acerca del comportamiento económico y social de los grupos humanos.

En segundo lugar, y con prioridad a las anteriores consideraciones, el espacio geográfico aparece organizado, "producido", por una sociedad determinada; es, en definitiva, un producto social «que materializa la capacidad tecnológica y productiva, los valores culturales e ideológicos dominantes, el sistema jurídico... es decir las características y lógica interna peculiares de una determinada sociedad en una etapa de su desarrollo histórico" (Méndez, 1988). Según esta perspectiva, el análisis que la Geografía realiza del espacio busca conocer, procura establecer, el con- 
junto de agentes sociales y la forma en que se desenvuelve la actuación de los mismos en la utilización y organización del territorio.

El interés cada vez mayor de los estudios del medio natural en poner de manifiesto determinadas prácticas que están agudizando la degradación del ambiente, la aplicación en el conocimiento de los factores que determinan la jerarquía urbana de un territorio, o las elaboraciones que arrojan luz acerca de la estratificación económica y social que puede apreciarse en la configuración interna de los núcleos urbanos... son algunos ejemplos de los numerosos trabajos geográficos en los que, cada vez en mayores proporciones, se rebasan explicaciones de mera causalidad material, interrogándose por los agentes sociales que realizan o fomentan determinadas prácticas de organización espacial.

Entendemos que, en el presente, los trabajos que buscan analizar un espacio concreto, implican el reconocimiento de todas las variables, tanto de las pertenecientes al medio natural como de aquellas que hacen referencia al medio socio-económico, que forman parte del espacio que se estudia bien por estar inscritos en él, bien porque organizan y configuran el espacio estudiado; implica, igualmente, una valoración de los mismos con indicación cualitativa y cuantitativa de su importancia, como paso previo para el establecimiento de un diagnóstico acerca de la dinámica y de los problemas que le afectan.

Hasta aquí hemos querido exponer brevemente aquellos elementos que se derivan de la particular atención que la Geografía ha prestado y presta al conocimiento de la dimensión espacial de hechos y fenómenos que en gran medida definen el quehacer del geógrafo.

Desde la perspectiva que nos ocupa nos parece conveniente resaltar la función específica que realizan las áreas de conocimiento que integran los estudios geográficos. Corresponde al área de Geografía Física el interés y atención por la distribución y comportamiento espacial de aspectos relativos al medio físico; el geógrafo interesado por las condiciones del medio natural puede trabajar una determinada particularidad del mismo, estudiando la forma bajo la que se presenta en diferentes lugares o bien establecer las peculiaridades de alguna o de la totalidad de las variables naturales correspondientes a un territorio concreto. De la misma manera, las distintas especialidades presentes en el área de Geografía Humana se fijan en la distribución espacial de determinadas características socioeconómicas o establecen las que corresponden a un espacio concreto. El área de Análisis Geográfico Regional se ocupa, de determinar y valorar todos los elementos, físico y socioeconómicos, presentes en un área espacial concreta sea cual sea la escala de trabajo adoptada. 


\subsubsection{La Geografía como Ciencia de interacciones}

No creemos incurrir en un error si afirmamos que la concepción de la Geografía como ciencia de interacciones entre hechos y fenómenos es una concepción muy querida y grata, aunque no exenta de problemas, para una parte importante de la comunidad de geógrafos; es, asimismo, una concepción que viene avalada por la tradición y que además se deduce con claridad de la propia consideración de la Geografia como disciplina ocupada en el análisis del espacio terrestre.

Nuestra formación en Geografía adquiere pleno sentido cuando concebimos los distintos aspectos que estudiamos no en sí mismos sino como hechos y fenómenos mutuamente interrelacionados.

La fuerza del geógrafo, su originalidad, la plenitud y satisfacción en el trabajo que ejecuta, radica en su voluntad integradora que tiende a relacionar, a establecer los nexos que existen en la realidad entre hechos estudiados fragmentariamente por diversas disciplinas científicas. Es en ello donde personalmente encuentro lo más valioso de la formación que he recibido $y$ en consecuencia lo que deseo y trato de comunicar en mi labor como profesor universitario.

No ignoramos las dificultades, más metodológicas que conceptuales, que entraña este enfoque de la Geografía; pero estas diicultades no son comparables a la desazón, intranquilidad, frustracción y falta de identidad que se produce en el geógrafo cuando, al perder esta visión, intenta emular las realizaciones de otros campos particulares de las ciencias. Por el contrario, lo genuino de la formación geográfica se entiende perfectamente cuando se conciben los distintos términos que estudiamos formando parte de una dinámica en la que los objetos aparecen no como elementos aislados sino reflejando el sistema de interacciones que tiene lugar en la realidad; porque lo que define el espacio terrestre, cualquier territorio, no es la simple adición de objetos, hechos y fenómenos en él contenidos, sino el complejo de interacciones entre esos objetos, hechos y fenómenos. Comprendemos bien esta afirmación si tenemos en cuenta que:

- Cualquier elemento presente en un espacio aparece influenciado por el resto de elementos que conjuntamente aparecen con él, de tal forma que la pregunta acerca de las propiedades, forma, causas o dinámica evolutiva del elemento considerado no puede obviar la apreciación del resto de elementos con los que se manifiesta y está asociado. 
- Las características del espacio terrestre vienen determinadas por el conjunto de flujos económicos, políticos, técnicos... que mantienen a diferentes escalas el conjunto de agentes que lo conforman $y$ organizan. El estudio de este tipo de flujos nos permite acercarnos al conocimiento de situaciones de predominio o de dependencia que afecta tanto al espacio en sí mismo considerado cuanto a los componentes sociales del mismo.

En consonancia con estas aseveraciones, la interpretación que la Geografía hace de un espacio determinado ya sea internacional, nacional, regional o local implica el reconocimiento de todas las variables que forman parte del mismo así como una valoración explícita de los nexos que entre dichas variables existen.

En este sentido, el sistema de correspondencias que obran en el interior del espacio terrestre y que son definidas en los trabajos geográficos hacen referencia:

- Al estudio de los procesos de interacción existentes entre los elementos constitutivos del medio natural de un espacio concreto;

- a la determinación de las influencias que pueden reconocerse entre los distintos componentes del ámbito social y económico de un espacio, así como de los que se establecen entre los agentes sociales que intervienen en la ordenación del espacio;

- como tema más específico, ligado a la tradición geográfica, al análisis de las relaciones e ilaciones mutuas entre el medio natural y el medio socioeconómico que conforman las señas de identidad de un espacio;

- al conocimiento del papel específico que desempeña un espacio en las relaciones de dominación o dependencia y de las consecuencias que de ello se derivan para la situación social y económica del mismo.

En esta concepción, no resulta difícil establecer las afinidades de cada uno de estos aspectos con el interés de las tres áreas de conocimiento que engloba la Geografía. Atañe a la Geografía Fisica establecer las características de un espacio desde la óptica de la dinámica y funcionamiento de los elementos naturales que lo constituyen mientras que en Geografía Humana el foco de atención profundiza en el estudio de las numerosas variables que, coaligadas, califican la situación social y económica de un territorio. Por su parte, la conceptualización que realiza el área de Análisis Geográfico Regional tiende a la interpretación del sistema territorial como sistema de relaciones entre el medio natural y el 
medio socioeconómico. Ninguna de las tres áreas puede desdeñar las repercusiones que los cambios producidos en un nivel concreto de la escala espacial tienen sobre el resto de los niveles escalares.

Completamos las presentes reflexiones indicando que, a nuestro juicio, la visión integradora de los estudios geográficos es compatible con la especialización que hoy advertimos en nuestra Ciencia, en la medida en que los distintos temas de estudio que abarcan estas especialidades, geografía rural, urbana... son resultado de la previa selección de un conjunto de fenómenos reconocidos como de particular incidencia en la organización espacial y no deben implicar ni el rechazo, ni el olvido del resto de elementos espaciales. En este sentido, el no perder la variedad de temas abordados en la formación geográfica constituye la mejor garantía para mantener y potenciar el sentido de integración, especialmente en aquéllos trabajos que tienden a nuclearse en torno a temas específicos.

\subsection{El papel de la Geografía Regional}

Hemos mencionado que en nuestra opinión compete al área de Geografía Regional definir la situación y dinámica que caracteriza a un territorio determinado mediante el análisis, reconocimiento y valoración de los elementos que lo forman y de las relaciones que entre ellos se establecen.

En este sentido la Geografía Regional atiende enteramente a las dos dimensiones que han sido objeto de atención preferente en los estudios geográficos: la dimensión espacial al estudiar un territorio concreto; la dimensión como ciencia de relaciones por cuanto en el análisis que hace de este espacio busca establecer las conexiones entre los elementos que lo integran y en especial las existentes entre medio físico y medio socioeconómico.

El trabajo geográfico regional requiere, según esto, reconocer el variado y complejo conjunto de elementos físicos, humanos y económicos que componen un espacio, examinar sus caracteristicas y peculiaridades integrándolos en un sistema de relaciones que nos permita comprender la estructura y dinámica del espacio considerado, las fuerzas que actúan y conforman su situación actual o que lo encaminan hacia una determinada situación futura. 
La finalidad que con ello se pretende puede ser variada; desde el afán por conocer una parte de la superficie terrestre por el puro placer de conocerla, hasta un tipo de conocimiento de las distintas zonas del espacio terrestre que permita establecer estrategias de actuación favorecedoras de intereses individuales o colectivos. Personalmente buscamos en nuestro trabajo el fomento de iniciativas que conduzcan a un aumento del desarrollo y del bienestar de las generaciones actuales y futuras asentadas en el espacio que estudiamos.

Sería ilógico pensar que una tarea de esta envergadura puede llevarse a cabo tomando como base las coordenadas que utiliza la Geografía Física en el tratamiento de los elementos del medio natural o aquellas en que se desenvuelve la Geografia Humana al ocuparse de los aspectos sociales y económicos. Entendemos que el punto en el que se sitúa el análisis que efectúa la Geografía Regional es aquel en el que hechos y fenómenos correspondientes a los elementos de cada uno de estos medios son considerados únicamente en los aspectos que pueden suponer una incidencia, tanto en el medio ambiente natural como en las características sociales y económicas del espacio concreto.

\subsection{Aspectos metodológicos de la Ciencia Geográfica}

Junto a los aspectos conceptuales, el plan de ordenación docente debe contener materias que satisfagan las necesidades metodológicas para el desarrolio del geógrafo.

La pregunta acerca de si el método empleado por la Geografía para llegar a comprender los conocimientos que le son propios es un método científico, ha suscitado una polémica tan viva como la del objeto de la Geografía. Es una polémica extendida a la totalidad de las ciencias sociales que, al estudiar el comportamiento humano o aspectos relacionados con el comportamiento humano, encuentran difícil establecer leyes universales al estilo de las ciencias físicas (Bunge, 1969 y 1980).

La defensa del carácter nomotético y del carácter idiográfico en el método científico utilizado por la Geografia, resume dos posturas clásicas y enfrentadas en esta materia que, en la actualidad, se intentan superar mediante la Teoría General de Sistemas. La incorporación en el Plan de Ordenación Docente de materias que permitan al estudiante abordar con solidez el conocimiento de estas técnicas de trabajo es una tarea sustancial para la futura formación geográfica. 


\section{ESTUDIOS GEOGRÁFICOS Y DEMANDA SOCIAL}

Uno de los problemas, posiblemente el principal, que hoy se plantea la comunidad universitaria es la integración en el mundo del trabajo de los profesionales que forma. En España son ya varias las generaciones de graduados que, acabados sus estudios, no encuentran lugar de trabajo y cuando lo encuentran es para dedicarse a tareas distintas de las que han ocupado su formación.

Afortunadamente los estudios geográficos pueden cubrir una variada gama de servicios requeridos por la sociedad. Una parte sustancial de esta demanda está relacionada con los requerimientos de la Administración para los trabajos de ordenación territorial a diversas escalas; necesidades que van desde la elaboración de una información cartogrática y temática básica para el reconocimiento de un territorio hasta la realización de análisis territoriales regionales, comarcales o locales, pasando por la elaboración de informes en materias específicas que tengan una clara proyección espacial.

Otra parte de la demanda se corresponde con la elaboración de monografias y estudios destinados a satisfacer los requerimientos del creciente público interesado en el conocimiento de su propio espacio o bien satisfacer las necesidades de empresas y organismos, tanto públicos como privados, que necesitan asesoramiento en materias tradicionalmente estudiadas por los geógrafos.

Un tercer tipo de demanda, que aunque tradicional no es conveniente minusvalorar porque de ella depende la compresión del interés social de la Geografia por parte de las futuras generaciones, debe cubrir las exigencias de formación de profesorado para impartir adecuadamente clases en los distintos niveles de enseñanza no universitaria.

Una última demanda se deriva de la necesaria formación científica e investigadora que solicita la Universidad y los centros de Investigación.

\subsection{Geografía y ordenación territorial}

"Cualquier concepción que trate de dar una visión holística de una serie de fenómenos complejos y dispersos recalará de alguna manera en la Ordenación del Territorio" (Leal Maldonado, 1988). Esta afirmación es expresión clara de que los estudios de planificación y ordenación territo- 
rial han podido, pueden y deben ser reconocidos como un campo apropiado de trabajo para el geógrafo; el análisis geográfico es prioritariamente análisis espacial que busca, comprende y explica las características de un territorio y que por ello está en condiciones de establecer las pautas de actuación para el desarrollo futuro del mismo. La preparación para tratar las distintas variables que conforman un espacio y su sentido integrador hacen del geógrafo un profesional válido y apto en tareas de ordenación territorial.

Solamente el juego político de intereses académicos, el escaso reconocimiento social de los estudios integrados en Facultades de Letras que son catalogados como "no técnicos" en un mundo fundamentalmente tecnificado y la dejadez, unas veces consciente, otras inconsciente, de los propios geógrafos que han considerado la enseñanza como el único objetivo de su formación, ha relegado y postergado las potencialidades y aptitudes que en esta materia proporcionan los estudios geográficos.

\subsubsection{Aportaciones de la Geografía en los análisis de Ordenación Territorial}

La Geografía puede aportar gran parte de lo que se considera necesario en este tipo de trabajos.

En primer lugar el reconocimiento del espacio sobre el que se quiere actuar, lo que significa poner de manifiesto sus componentes, su estado actual y su evolución histórica, las relaciones entre ellos, así como la dinámica evolutiva que les afecta en su individualidad y como conjunto y los agentes que han controlado y controlan los procesos de transformación.

En segundo lugar la capacidad para establecer un diagnóstico que indique cuál es la situación, la intensidad en el aprovechamiento de los recursos disponibles y los espacios de fragilidad.

En tercer lugar la aptitud para valorar y graduar los problemas del territorio en cuestión y para proponer soluciones y alternativas que permitan mejorar y conseguir los objetivos anteriormente dichos sin agredir formas de vida que constituyen auténticos patrimonios culturales.

El geógrafo aporta la visión conjunta de todos estos pasos y de lo exigido por cada uno de ellos; visión que normalmente está ausente de las personas que componen los equipos interdisciplinares en cuyo seno 
deben realizarse los planes de ordenación territorial porque en muchas ocasiones tanto los elementos de un espacio como los procesos a que están sometidos requieren conocimientos que no son específicos de la especialidad geográfica.

Por último, la ordenación del territorio responde a un conjunto de actos administrativos y conlleva la cumplimentación de determinados aspectos formales así como el conocimiento, o al menos la toma en consideración, de cuales son las posibles vías de financiación que permitan la realización de las acciones que se plantean en el trabajo realizado.

Los cuatro puntos mencionados como esenciales en todo trabajo de ordenación territorial, no agotan la complejidad de las actuaciones. Tan importante como la tarea de reconocimiento, interpretación y valoración de hechos, diagnósticos y presentación de alternativas, es la manera en que se lleva a cabo, cómo se realiza y organiza el propio proceso de planificación territorial. Merece la pena que dediquemos algunas reflexiones a este tema.

En Geografía solemos decir que la labor de despacho debe estar avalada y acompañada del trabajo de campo; lo mismo cabe indicar para los análisis de ordenación territorial.

Por su propia naturaleza las acciones sobre el territorio van simpre dirigidas y afectan a los hombres que viven en un determinado espacio. A nuestro juicio, gran parte de las deficiencias observables en las actuaciones de planificación responden a la inadecuación entre la visión resultante de los planteamientos teóricos sobre la problemática del espacio que se estudia y la visión real de las personas a quien afecta o de los agentes que se desea actúen para modificar una situación; en numerosas ocasiones se generalizan medidas de desarrollo que si bien son aptas y dan fruto a determinadas escalas o en determinados lugares, no son aprovechables en otros porque los incentivos, aún siendo atractivos, no responden a las expectativas globales de los habitantes de ese espacio. A ello se une un planteamiento demasiado rígido, que impide modificar las medidas de actuación previamente programadas.

Somos de la opinión que el modelo de organización en los procesos de ordenación territorial debe ir de abajo a arriba, de forma que en todos sus pasos implique y comprometa a las personas afectadas; el reto, que en este sentido, tienen planteados las diferentes Administraciones acostumbradas a una actuación inversa es sin lugar a dudas importante.

Particularmente considero muy a tener en cuenta estas ideas cuando la labor de ordenación se centra en áreas comarcales o locales, sobre 
todo dentro del actual contexto que pone en el desarrollo autogenerado y en el aprovechamiento de los recursos locales las bases fundamentales para iniciar una dinámica de cambio.

En estas áreas se necesitan personas, profesionales, que en el trabajo de ordenación sepan recoger la visión de los habitantes, imprimir en ellos la conciencia de que se puede modificar una situación, agrupar esfuerzos y conocer con qué medios se puede contar para desarrollar ese espacio. Profesionales que podríamos denominar Agentes de Ordenación que serían auténticos animadores de los procesos de planificación establecidos en una zona. Tendrían como función la realización de todos los pasos que requieren las tareas de ordenación territorial con los habitantes de un territorio. Estos profesionales necesitarían un tipo de información perfectamente asimilable en el plan de estudios de Geografía, respondiendo a una demanda que se está haciendo sentir en numerosos paises.

\subsection{Otras demandas no específicamente docentes}

Si los análisis de ordenación territorial forman un ámbito de trabajo en el que muy bien pueden desenvolverse los profesionales geógrafos, existen igualmente un conjunto de demandas, con un cierto grado de indeterminación y variabilidad, pero que aumentan constantemente a medida que se incrementa el nivel y calidad de vida de los ciudadanos. Nos referimos a los trabajos que tienden a cubrir el interés de las personas por conocer las características de su propio territorio o de territorios lejanos así como las necesidades de instituciones públicas en materia de cartografía temática.

La divulgación de temas referidos a la propia región o a espacios comarcales de la misma; la redacción de guias, itinerarios naturalísticos o paisajísticos, urbanos, comarcales o regionales, que invitan a visitar el territorio sobre el que se vive y se apoyan la educación medioambiental, los informes que plasman la problemática económica y social, los modos y formas de vida, las condiciones naturales de distintos países del mundo, son aspectos que siempre han encontrado amplio eco y demanda en la sociedad pero que se hacen más perentorios cuando se sobrepasan determinados niveles de renta y bienestar. En la actualidad las posibilidades técnicas y de difusión de los medios de comunicación, el interés de los gobiernos autonómicos y locales por dar a conocer estos temas, la propia industria editorial que encuentra extenso mercado en 
estas obras, favorecen la demanda de este tipo de trabajos que han sido tratados tradicionalmente en la literatura y obras geográficas. La especialidad de Geografía puede perfectamente preparar profesionales aptos para estos menesteres.

Por otra parte, el mapa, la cartografía como expresión visual y gráfica de los hechos geográficos es consustancial al quehacer del geógrafo. En todos los planes de estudio se ha hecho especial hincapié en la interpretación y elaboración de una cartografía temática adecuada. La Administración, tanto autonómica como local, necesitan disponer para sus actuaciones en el territorio de un material cartográfico de base, algunos de cuyos temas (usos de suelo, cultivos y aprovechamientos, mapas específicos de determinados aspectos..) pueden ser confeccionados por geógrafos.

\subsection{Geografía y enseñanza en niveles no universitarios}

La formación de profesores capaces de impartir Geografia en los distintos niveles de enseñanza no universitaria, ha sido una de las finalidades esenciales de los programas que rigen en los Departamentos actuales de la especialidad.

La disminución del número de alumnos que progresivamente se escolarizan, consecuencia de los cambios que han tenido lugar en el comportamiento demográfico de la sociedad, no puede suponer insensibilidad hacia una labor que siempre será primordial. Por ello debe mantenerse un plan de estudios específico dirigido a la formación de profesores que integre, junto a las materias propias de la especialidad, materias afines que como la historia, la sociología o la economía permitan adquirir una cultura general, no reñida con la calidad, con la suficiente flexibilidad que, en cuanto a conocimientos, demandan de los profesores los centros de enseñanza.

\subsection{Geografía, enseñanza e investigación universitaria}

La Ley de Reforma Universitaria y los Estatutos de las distintas Universidades regulan en España el acceso a las tareas docentes e investigadoras de la Universidad. Los estudios de Tercer ciclo y las funciones específicas que la ley encomienda a los Profesores Ayudantes conforman 
la formación inmediata de las personas que se van a integrar en un Departamento Universitario.

Los estudios de Tercer Ciclo forman parte de los planes de estudio de un Departamento y administrativamente tienden al reconocimiento de la suficiencia investigadora o lo que es lo mismo al reconocimiento de la capacidad para realizar trabajos de investigación en centros públicos y privados.

El decreto que regula estos estudios (RD 185/1985 de 23 de enero; BOE, 16-11-1985) establece la necesidad de obtener una serie de créditos en materias conceptuales (doce al menos) y otros en materias metodológicas. La adaptación de los Departamentos a la nueva normativa ha multiplicado considerablemente la oferta, anteriormente muy limitada, de cursos de Doctorado en Geografía, beneficiando al alumnado que, teóricamente, puede elegir según sus gustos y especialización curricular.

Sin embargo, el programa de Tercer Ciclo de un Departamento debe ser algo más que un agregado de cursos ofertados en función de las líneas de investigación que particularmente siguen cada uno de sus miembros y pasar a convertirse en uno o varios programas dotados de coherencia y unidad interna. Es posible organizar estos estudios en torno a núcleos o centros de interés como pueden ser el urbanismo, el medio rural, los recursos hídricos, la actividad agraria... e integrar en torno a ellos el análisis especializado de las personas del Departamento. Para ello se hace imprescindible, o por lo menos la lógica así nos to hace pensar, que el Departamento tenga unas líneas de investigación propias con objetivos a medio plazo, elaborados y consensuados por todos sus miembros en vez de la fragmentación y dispersión de esfuerzos actualmente en uso.

Compete al Departamento velar y crear las condiciones necesarias para la formación de Profesores Ayudantes; de ello va a depender la futura calidad y continuidad del propio Departamento. Han pasado ya los tiempos en que el Profesor Ayudante era el encargado de cubrir los huecos de una deficiente dotación de personal de servicios $y$, al menos en la realidad actual tiene clara conciencia del periodo de formación en que se encuentran las personas que realizan esta función.

Bajo la tutela de un profesor, normalmente director del trabajo de tesis, tienen a su cargo la realización de actividades complementarias concebidas, planificadas y realizadas bajo la supervisión del Profesor titular de la asignatura correspondiente, y la impartición de un número reducido y progresivo de clases teóricas. 
Participar con los distintos profesores del Departamento en la elaboración de programas, preparación de algunas clases teóricas, proyectos de investigación..., así como facilitar al Profesor Ayudante los medios materiales para acudir a cursos de perfeccionamiento intercambios y estancias en otras Universidades, es algo necesario que redundará ineludiblemente en beneficio del Departamento.

\section{ORDENACIÓN DOCENTE EN LOS ESTUDIOS DE GEOGRAFÍA REGIONAL}

Establecido el marco conceptual y metodológico de la Ciencia geográfica y las demandas que pueden ser satisfechas mediante un estudio, nos concentramos a continuación en la exposición de un posible plan de estudios.

Las propuestas ministeriales sobre el futuro de las enseñanzas universitarias en España contempla el establecimiento de dos ciclos en la carrera universitaria. La novedad con respecto a lo actualmente establecido estriba en la posibilidad de obtener un título terminal (Diplomado en Geografía y Ordenación Territorial) al acabar los 180 créditos que aproximadamente tiene asignado el primer ciclo de enseñanzas universitarias (1.800 horas lectivas). La idea, en principio, no ha tenido excesiva contestación en la comunidad de geógrafos españoles; sin embargo, la validez de la misma está relacionada y depende de las funciones, hasta el momento no claramente especificadas, que se asignen a este título de Diplomatura.

\subsection{El sentido de los estudios de Diplomatura}

En nuestra opinión, el carácter de los estudios de primer ciclo como requisito imprescindible para cursar la Licenciatura, indica con claridad que se trata de proporcionar un aprendizaje conceptual y metodológico básico, sustancial; el fundamento, en definitiva, de lo que constituye la formación de un geógrafo. La afirmación de este carácter nos parece de sumo interés porque de ella deducimos un período de enseñanza desprendido de las rigideces a que conduce la especialización en áreas de conocimiento o en determinadas materias dentro de estas áreas. 
Entendemos, pues, la ordenación de los estudios en un primer ciclo de formación general, lo que no significa superficial, que proporcione los conceptos y metodología básicos en los estudios espaciales y un segundo ciclo de especialización en una de las tres áreas; esta ordenación permitiría, entre otras cosas, profundizar en materias especializadas desde una perspectiva geográfica y no desde la perspectiva de ciencias afines y volver a acariciar la idea, que en numerosas ocasiones oimos y que compartimos, de ser, ante todo geógrafos. Al mismo tiempo, si tomamos en consideración la nominación más admitida en las propuestas enviadas al Consejo de Universidades, "Diplomado en Geografía y Análisis Territorial”, y los objetivos que en ellas se formulan para el primer ciclo se desprende una clara orientación hacia las acciones de ordenación territorial.

El sentido básico de los estudios de Geografía, sin más adjetivos, y la realización de trabajos en el campo de la ordenación territorial, una vez que hemos manifestado cuáles son las ideas que pensamos esenciales en ambas materias, son dos claves que nos permiten responder a la cuestión planteada y diseñar las funciones operativas específicas de este ciclo. Serían las siguientes:

- Profesionales con un alto grado de conocimiento y capacidad de valoración de las fuentes de documentación geográfica. Es una función de suma importancia en las Administraciones Públicas que necesitan de un buen banco de datos continuamente actualizado y fiable para proyectar, programar y realizar coherentemente las actuaciones sobre el territorio.

- Expertos en la elaboración de cartografía temática que recoja aquellos elementos de particular significación espacial, tanto desde una perspectiva estática como dinámica, y elabore los informes correspondientes. Esta función es la lógica consecuencia del interés tradicional de los trabajos geográficos por la plasmación cartográfica de las distribuciones espaciales de hechos y fenómenos.

- Personal cualificado en la confección de informes que requieran poner de manifiesto las interacciones medioambientales, socioeconómicas o globales en un espacio concreto.

- Diplomados expertos en conocimientos regionales, juzgando como tales no solamente los que poseen un alto grado de saber acerca de la situación de espacios concretos a distintas escalas, sino la formación de personas capaces de llevar a buen puerto las accio- 
nes de ordenación territorial que se propugnan para un espacio regional, comarcal o local.

\subsection{Estudios de Diplomatura en Geografía y Análisis Territorial}

Las materias que creemos deben formar parte de los estudios de Diplomatura en Geografía y Análisis Territorial, no propias del área de Análisis Geográfico Regional, podemos agruparlas en cuatro grandes bloques temáticos:

- Materias cuya finalidad didáctica tiende al conocimiento y valoración de los elementos que forman parte del medio natural como fuente de recursos varios para la humanidad, así como de la dinámica y relaciones presentes en el complejo físico de un espacio.

- Materias cuya finalidad didáctica tiende al conocimiento y valoración de las variables socio-económicas y la forma en que dichas variables interactúan a nivel espacial.

- Materias encaminadas a la toma de conocimientos relativos a las fuentes de información necesarias para el análisis de las variables, del medio natural y del ámbito económico-social y aquellas que suministran los medios técnicos para la información.

- Materias enfocadas hacia el conocimiento del funcionamiento y sistemas del planeamiento de las Administraciones territoriales.

El área de Análisis Geográfico Regional está presente en los estudios de Diplomatura mediante la impartición de las materias que se ocupan de ámbitos espaciales definidos. El conocimiento de los temas a que da acceso el estudio de las materias de esta área es fundamental en la formación del geógrafo. Primero, porque las materias sobre espacios regionales tienen un inequívoco sentido referencial, son imprescindibles para enmarcar espacialmente los hechos y fenómenos que se estudian en otras materias. Segundo, porque permiten establecer rasgos comparativos entre las distintas zonas de la Tierra y entre los caminos que han seguido y siguen los espacios regionales en sus políticas y medios de ordenación territorial. Tercero, y más significativo, porque bien concebidas son un poderoso instrumento para crear el hábito de correlacionar los elementos que organizan el espacio, aspecto esencial en toda labor geográfica que adquiere en la concepción regional su más alta expresión; acostumbran al alumno a interpretar esa realidad que las ciencias analíticas presentan desde diversos ángulos pero siempre desde una óptica 
parcial y a ver los fenómenos como realmente se dan, dentro de un todo orgánico. Aún podemos añadir un último punto, compartido con otros muchos estudios, referido al caudal de erudición que los estudios regionales permiten adquirir.

\subsection{Plan de estudios en la Diplomatura}

Las asignaturas que proponemos en los estudios de Diplomatura en función de lo anteriormente expresado son las siguientes:

\section{Primer Curso de Diplomatura}

Fundamentos de Geomorfología

Fundamento de Climatología e Hidrología

6 créditos

Historia Económica Contemporánea

6 créditos

Introducción a la Geografía Económica

Geografía de la Población y de los asentamientos

8 créditos

6 créditos

Análisis e interpretación del Mapa Topográfico

6 créditos

Análisis e interpretación de Mapas Temáticos 1

3 créditos

Obtención y valoración de Fuentes de Información numérica

2 créditos

Aplicaciones informáticas en Geografía I

3 créditos

6 créditos

Geografía Regional del mundo

14 créditos

\section{Segundo Curso de Diplomatura}

Fundamentos de Edafología y Biogeografía

Geografía de las Actividades Productivas

7 créditos

7 créditos

Análisis e interpretación de Mapas Temáticos II

2 créditos

Observación y valoración de Fuentes de

Información numérica II

3 créditos

6 créditos

Aplicaciones informáticas en Geografía II

Técnicas de trabajo de campo y laboratorio

5 créditos

Técnicas de Análisis de datos geográficos

Métodos gráficos para el tratamiento de la

Información Geográfica

10 créditos

Geografía de la Comunidad Económica Europea

Geografía de la Península Ibérica

4 créditos

7 créditos

9 créditos

\section{Tercer Curso de Diplomatura}

Paisajes naturales

8 créditos

Bases para el reconocimiento social y económico del Territorio

8 créditos 
Fotointerpretación

Introducción a la Teledetección

Elaboración de cartografía temática

Introducción a la dinámica de sistemas

Legislación y Administración Territorial

Gestión Territorial

Geografia Regional de España
10 créditos

4 créditos

8 créditos

4 créditos

5 créditos

4 créditos

9 créditos

\subsection{Los estudios de Licenciatura en Geografía y ordenación del territorio}

Al carácter de formación geográfica general que concebimos para los estudios de Diplomatura sucede en el tiempo la adquisición de conocimientos especificos y especializados de los cursos de Licenciatura. Con la formación básica que se supone adquirida en cursos anteriores, el alumno puede integrarse en el estudio de los conocimientos propios de las distintas subdisciplinas geográficas a las que prestan especial atención las diferentes áreas que identifican la Geografía actual.

Dos son los objetivos marcados para el ciclo de Licenciatura:

- De acuerdo con la denominación de la especialidad, formar personas preparadas para el ejercicio de los trabajos de ordenación y planificación territorial desde distintas ópticas y perspectivas: ordenación territorial del medio físico, ordenación y planificación urbana, ordenación regional...

- Sentar las bases para la formación y capacitación en tareas de investigación en las distintas materias que son objeto de la Geografia.

Lógicamente cada una de las áreas de conocimiento deberá establecer las asignaturas que crea convenientes para cumplir los objetivos fijados en la especialidad, sin que ello implique una dispersión de esfuerzos del conjunto del Departamento. En el caso del área de Geografía Regional pensamos que las materias de segundo ciclo deben encaminarse fundamentalmente hacia el conocimiento de los medios e instrumentos de una ordenación integral e integrada del espacio regional. 


\subsubsection{Asignaturas de interés para la formación geográfica en Licenciatura}

Los alumnos podrán completar la formación adquirida en los cursos de Diplomatura eligiendo aquellas asignaturas de entre las ofertadas por las distintas áreas de conocimiento, que crean, más conveniente a sus intereses formativos. Sin embargo, dentro de la línea de afianzamiento de los saberes teóricos e instrumentales ya adquiridos y por la orientación hacia la ordenación territorial que deseamos para los estudios geográficos, creemos se estará de acuerdo en establecer materias instrumentales comunes a las tres áreas que deberian ser seguidas por todos los alumnos. Entre estas materias proponemos las siguientes:

- Teledetección.

- Dinámica de Sistemas.

- Procedimientos generales para la elaboración de teorías y modelos.

- Teoría de la Geografía.

Competeria al área de Análisis Geográfico Regional tomar la responsabilidad y asumir la impartición de dos asignaturas específicas en los estudios de Licenciatura. Las dos asignaturas íntimamente ligadas a los trabajos de ordenación territorial.

La primera de ellas trataría la forma de abordar el análisis de todos los componentes que intervienen y han de ser tenidos en cuenta en los trabajos de ordenación territorial. Es una asignatura a la que creemos debe dedicarse una parte sustancial del tiempo de aprendizaje de los alumnos que deseen esta orientación; tiempo que ciframos en el correspondiente a treinta créditos de los cuales veinte se desarrollarían en el primer año de la Licenciatura y diez en el segundo año.

La segunda asignatura no lo es en el sentido estricto del término, puesto que proponemos la realización de un trabajo de ordenación en un área espacial concreta, con un tiempo de dedicación correspondiente a veinticinco créditos, a desarrollar durante el segundo y tercer trimestre del segundo año de Licenciatura. Dicho trabajo se realizaria bajo la dirección de uno o varios profesores del área, con sesiones periódicas de revisión en grupo de lo que se va realizando; sesiones que servirian igualmente para solventar las dudas y problemas que se planteen.

La realización de este trabajo permitiria aplicar las teorías y técnicas aprendidas en cursos anteriores e iniciaría a los alumnos en la resolución 
de problemas concretos con los que se van a topar en su ejercicio profesional. La tarea de recogida de información e incluso de una primera elaboración de la misma podría compaginarse con el desarrollo del curso teórico de ordenación territorial mencionado con anterioridad. Incluso sería factible el establecimiento de conciertos, con organismos prioritariamente administrativos, que apoyasen la realización de este tipo de estudios.

Al margen de estas dos asignaturas, el área de Análisis Geográfico Regional podría, en dependencia de los medios humanos disponibles, implantar otras asignaturas con carácter opcional en las que se profundizase en el conocimiento de áreas espaciales concretas, continentes, países o regiones, que ya fueron objeto de tratamiento en los cursos de Diplomatura.

Admito que puede resultar pretencioso para los profesionales geógrafos no adscritos al área de Análisis Geográfico Regional, la asignación de una asignatura específica sobre ordenación territorial a nuestra área de Análisis. Entre otras consideraciones, y no sin razón, se nos puede decir que las tareas de ordenación territorial tienen un carácter interdisciplinar y que en ellas han de intervenir, no ya sólo las distintas áreas de conocimiento geográfico, sino también otras disciplinas científicas ajenas al propio campo geográfico. Ello es indudablemente cierto por lo que la asignación de esta materia al área de Análisis Geográfico Regional no puede significar ni la exclusión de las otras áreas ni, menos todavía, la exclusión en su impartición de profesionales de otras áreas de conocimiento.

En realidad integramos en una asignatura, realmente voluminosa, aspectos que pueden ser tratados por asignaturas de otras áreas. ¿Porqué lo hacemos así? Varias razones nos mueven a adoptar esta postura:

- La primera mantener la coherencia con los postulados en los que hemos basado la especificidad del Análisis Geográfico Regional y el sentido de las tareas de ordenación territorial. Se trata de trabajar sobre espacios concretos y analizar el sistema de interacciones entre el medio natural y el medio socioeconómico, aspectos ambos inherentes al área de Análisis Geográfico Regional.

- En segundo lugar, tanto en el área de Geografía Física como en el área de Geografía Humana se realizan trabajos de indudable proyección en la ordenación territorial, pero en aspectos determinados y no como conjunto. Estas áreas poseen además, campos de trabajo que rebasan la orientación propia de los temas de ordenación. 
- Por último, creemos conveniente una formación específica en tareas de ordenación y ello requiere asignaturas orientadas específicamente a ello. La implantación de asignaturas optativas en los cursos de especialidad puede permitir que un alumno estudie temas concretos de ordenación territorial pero no todos. Una asignatura dedicada a ello evita las lagunas a que el sistema de asignaturas optativas puede dar lugar, aunque hemos de reconocer que sin duda alguna se producirán solapamientos y repeticiones en determinados contenidos que en cualquier caso no creemos afecten a un elevado número de alumnos. 


\section{BIBLIOGRAFÍA}

Estébanez Álvarez, J., 1982: “La enseñanza de la Geografía en la Universidad". Real Sociedad Geográfica, tomo CXVII, p. 179-199.

CAPEL, H., 1982: “Positivismo y antipositivismo en la Ciencia Geográfica. El ejemplo de la Geomorfologia». Actas I Congreso de Teoría y Metodología de las Ciencias. Oviedo, p. 256-260.

MÉnDEZ, R.: “El espacio de la Geografía Humana». Geografía Humana. Madrid. Ed. Cátedra, p. 10.

Bunge, M., 1969: La investigación científica. Barcelona. Ed. Ariel.

-1980: Epistemología. Barcelona. Ed. Ariel.

Leal Maldonado, J., 1988: "La Ordenación del Territorio en la encrucijada». Rev. Papeles de Economía Española. Fundación. Madrid. Fondo para la Investigación Económica y Social, p. 58. 